\title{
Conservation of the Lateral Electron Momentum at a Metal-Semiconductor Interface Studied by Ballistic Electron Emission Microscopy
}

\author{
C. A. Bobisch, ${ }^{1, *}$ A. Bannani, ${ }^{1}$ Yu. M. Koroteev, ${ }^{2}$ G. Bihlmayer, ${ }^{3}$ E. V. Chulkov, ${ }^{4,5}$ and R. Möller ${ }^{1}$ \\ ${ }^{1}$ Department of Physics, Center for Nanointegration Duisburg-Essen, University of Duisburg-Essen, 47048 Duisburg, Germany \\ ${ }^{2}$ ISPMS, RAS, 634021, Tomsk, Russia \\ ${ }^{3}$ Institut für Festkörperforschung, Forschungszentrum Jülich, D-52425 Jülich, Germany \\ ${ }^{4}$ Departamento de Fsica de Materiales and Centro Mixto CSIV-UPV/EHU, Facultad de Ciencias Qumicas, UPV/EHU, \\ Apartado 1072, 20080 San Sebastin, Basque Country, Spain \\ ${ }^{5}$ DIPC, 20018 San Sebastin/Donostia, Basque Country, Spain
}

(Received 15 December 2008; published 1 April 2009)

\begin{abstract}
We report on ballistic electron emission microscopy and spectroscopy studies on epitaxial (3-5 nm thick) Bi(111) films, grown on $n$-type Si substrates. The effective barrier heights of the Schottky barrier observed are $0.58 \mathrm{eV}$ for the $\mathrm{Bi} / \mathrm{Si}(100)-(2 \times 1)$ and $0.68 \mathrm{eV}$ for the $\mathrm{Bi} / \mathrm{Si}(111)-(7 \times 7)$. At the step edges of the epitaxial films a strong increase of the ballistic electron emission microscopy current is observed for $\mathrm{Bi} / \mathrm{Si}(111)-(7 \times 7)$, while no increase occurs for $\mathrm{Bi} / \mathrm{Si}(100)-(2 \times 1)$. These observations can be explained by the conservation of the lateral momentum of the electron at the metal-semiconductor interface.
\end{abstract}

DOI: 10.1103/PhysRevLett.102.136807

PACS numbers: 73.23.Ad, 73.20.At, 73.30.+y, 73.40.-c

Since the invention of ballistic electron emission microscopy (BEEM) by Bell and Kaiser two decades ago $[1,2]$, studies on the conservation of lateral electron momentum at the interface formed between metals and semiconductors have remained puzzling. Schowalter and Lee $[3,4]$ studied ballistic electron emission spectroscopy (BEES) for Au films grown on Si substrates with different crystallographic orientations. Assuming that the lateral electron momentum at the metal-semiconductor interface is conserved [2] the BEEM currents were expected to differ dramatically due to different projections of the conduction band minima of the $\mathrm{Si}$ on the (111) and (100) plane. However, almost identical BEEM currents were measured. These results were later confirmed by Weilmeier et al. [5,6]. In the case of Pd on Si, Ludeke and Bauer [7] attributed the observed equality of transmission across (111) and (100) to interface scattering randomizing the electron momentum. As discussed by García-Vidal et al. [8] and Bell [9], the results found on Au samples may be explained without additional electronic scattering processes, because the electronic band structure of the transition metals ( $\mathrm{Au}, \mathrm{Ag}, \mathrm{Pd}$, etc.) exhibits a band gap in the [111] direction, requiring a minimal lateral component of the electronic momentum. Taking into account the matching conditions at the metal-semiconductor interface, they predicted a similar onset for the BEEM current, but a higher intensity for $\mathrm{Au} / \mathrm{Si}(111)$ in agreement with the experiments.

A different situation is found with $\mathrm{CoSi}_{2} / \mathrm{Si}(100)$ versus $\mathrm{CoSi}_{2} / \mathrm{Si}(111)$. The band structure of $\mathrm{CoSi}_{2}$ allows the propagation of electrons at $k_{\|}=0$, and due to the excellent quality of the epitaxial layers there is very little diffuse scattering of electrons. The latter is confirmed by the high resolution of detail at the metal-semiconductor interface obtained by BEEM imaging [10], indicating that the electrons are limited to a very small cone within the $\mathrm{CoSi}_{2}$. A theoretical description by García-Vidal et al. [8,11-14] could explain the high spatial resolution for BEEM and has predicted that the onset for the BEEM current should be shifted for the (111) versus the (100) substrate, due to the conservation of the lateral momentum at the interface. However, the experimental results for the BEEM current are contradictory. While Kaiser et al. [15] found a delayed onset of the BEEM current for the (111) oriented versus the (100) oriented substrate, Sirringhaus et al. [10] did not find a significant difference.

As pointed out by de Pablos et al. [14], the lack of experimental results on well-characterized metal layers grown on semiconductor surfaces does not allow a final conclusion thus far. In this work, we present BEEM studies on epitaxial $\mathrm{Bi}(111)$ films grown on $\mathrm{Si}(100)-(2 \times 1)$ and $\mathrm{Si}(111)-(7 \times 7)$ substrates [the rhombohedral indexing for the $\operatorname{Bi}(111)$ surface will be used in this Letter]. In a previous study on $\mathrm{Bi} / \mathrm{Si}(100)$ we reported on a long range modulation of the BEEM signal due to transport into different oriented substrate terraces [16]. In contrast to the noble metals, the electronic band structure of the $\mathrm{Bi}$ allows the injection of hot electrons near $k_{\|}=0$ [17-19]. Moreover, epitaxial $\mathrm{Bi}(111)$ films can be grown with minimal roughness at the interface and the surface. Our results clearly reveal a delayed onset of the BEEM current for the (111) orientation of the substrate. Combined with data obtained at step edges, this yields strong evidence that the lateral momentum is conserved at the interface.

The experiments were performed in a commercial ultrahigh vacuum multiprobe STM at $130 \mathrm{~K}$. The surface of $n$-type $\mathrm{Si}$ samples with resistivities between $1-30 \Omega \mathrm{cm}$ and an Ohmic back contact was prepared in situ by flashing 
the substrate to $1500 \mathrm{~K}$ several times. An amount of $3-5 \mathrm{~nm}$ of $\mathrm{Bi}$, corresponding to $8-13 \mathrm{Bi}$ bilayer, was deposited at a rate of about $0.15 \mathrm{~nm} / \mathrm{min}$ onto the cooled sample surface $\left(T_{\text {sample }}=130 \mathrm{~K}\right)$. The samples were moderately annealed to room temperature, resulting in epitaxial Bi(111) films [20], as was checked by low energy electron diffraction (LEED).

The BEES results are displayed in Fig. 1. The data were acquired at different points on the sample as well as on different samples; at each point several spectra were measured. Each spectrum was fitted to $I_{\text {BEEM }} \propto$ $R\left[\left(E-\Phi_{B}\right)^{2} / E\right][21]$ up to about twice the expected barrier height $\Phi_{B}(R$ is the transmission factor).

A representative spectrum for $\mathrm{Bi} / \mathrm{Si}(100)-(2 \times 1)$ is shown in Fig. 1(a). The evaluation of 60 numerical fits yields a mean value of $(0.58 \pm 0.04) \mathrm{eV}$ for the Schottky barrier height [Fig. 1(b)]. As can be seen in the spectrum in 1(a), the yield of the BEEM electrons is remarkably high, compared to values reported in the literature [22-24] for other metal-semiconductor interfaces.

The results for $\mathrm{Bi} / \mathrm{Si}(111)-(7 \times 7)$ are shown in Figs. 1(c) and 1(d). Forty-eight spectra were fitted, yielding a mean apparent barrier height of $(0.68 \pm 0.03) \mathrm{eV}$. Hence, the onset for the ballistic transmission is offset by $(0.10 \pm 0.05) \mathrm{eV}$ to higher energies. The BEEM current is high also for $\mathrm{Bi} / \mathrm{Si}(100)-(2 \times 1)$. The ratio of the prefactors $R_{\mathrm{Si}(100)} / R_{\mathrm{Si}(111)}=1.23$ indicates that the ballistic
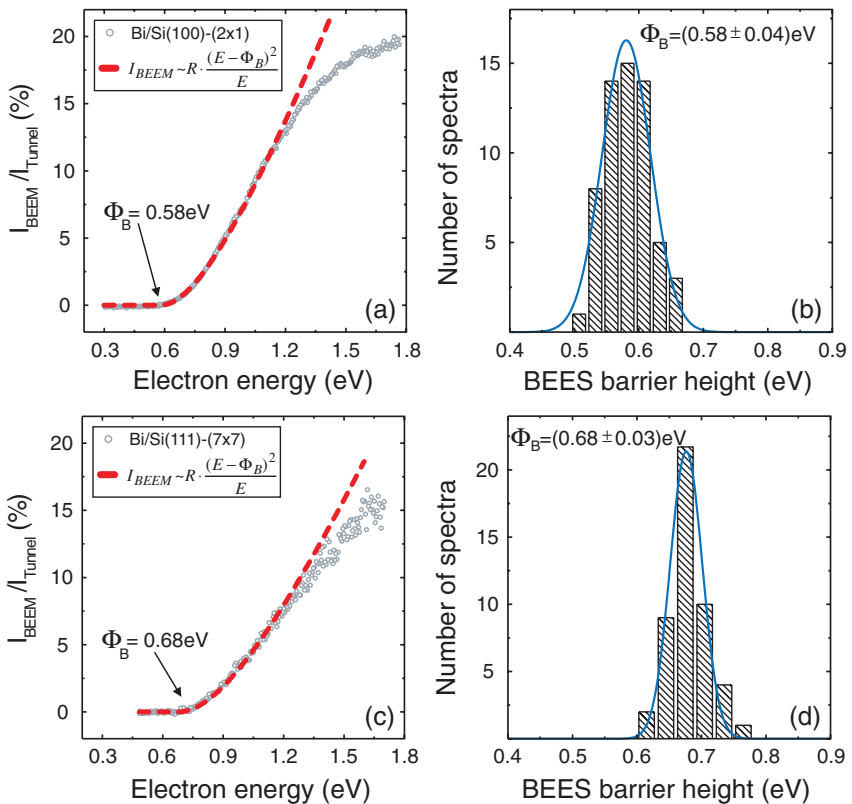

FIG. 1 (color online). (a) BEES spectrum for $\mathrm{Bi}(111)$ $\operatorname{Si}(100)-(2 \times 1)$, measured at $I_{\text {tunnel }}=40 \mathrm{pA}$. The numerical fit yields a local Schottky barrier height of $0.58 \mathrm{eV}$. (b) Distribution of Schottky barrier heights for 60 spectra. (c) BEES spectrum, measured on $\mathrm{Bi}(111)-\mathrm{Si}(111)-(7 \times 7)$ at $I_{\text {tunnel }}=82 \mathrm{pA}$. The fit yields a local Schottky barrier height of $0.68 \mathrm{eV}$. (d) Distribution of Schottky barrier heights for 48 spectra. transmission is somewhat more efficient for the $\mathrm{Bi} / \mathrm{Si}(100)-(2 \times 1)$ interface.

The results obtained with BEEM for $\mathrm{Bi} / \mathrm{Si}(100)$ are shown in Fig. 2. The topography in 2(a) shows a highly crystalline, flat and smooth film; triangular shaped holes with a depth of one Bi bilayer are visible. The slight undulation in the topography originates from overgrown substrate terraces, due to a miscut of $\approx 1^{\circ}$ of the $\mathrm{Si}(100)$ wafer. In spite of the different types of symmetry, threefold for the $\operatorname{Bi}(111)$ and twofold for $\operatorname{Si}(100)-(2 \times 1)$, both lattices can be matched commensurably [20,25]. Individual domains can grow over several substrate steps without being affected [16]. In the lower right-hand part of the image a mirror domain is visible, changing the characteristic orientation of the triangular shaped holes by $180^{\circ}$. The domain boundary is indicated by the dashed white line.

The simultaneously measured BEEM current at an injection energy of about $1.8 \mathrm{eV}$ is displayed in Fig. 2(b). The image shows an alternating pattern of dark and bright stripes, with an average BEEM current of $8.5 \%$ of the tunneling current in the dark and $10.5 \%$ in the bright areas, respectively. This may be attributed to the $(2 \times 1)$ and the $(1 \times 2)$ reconstruction of the $\operatorname{Si}(100)$ surface. The observations reported in [16] indicate that the $(2 \times 1)$ reconstruction may be maintained underneath the Bi layer. A close view of the data for a triangular hole is displayed in 2(c) and 2(d). It reveals a faint variation of the BEEM current at the step edges. However, the data for the reverse direction displayed in 2(e) show the opposite contrast, indicating an artifact of the measurement. Obviously the

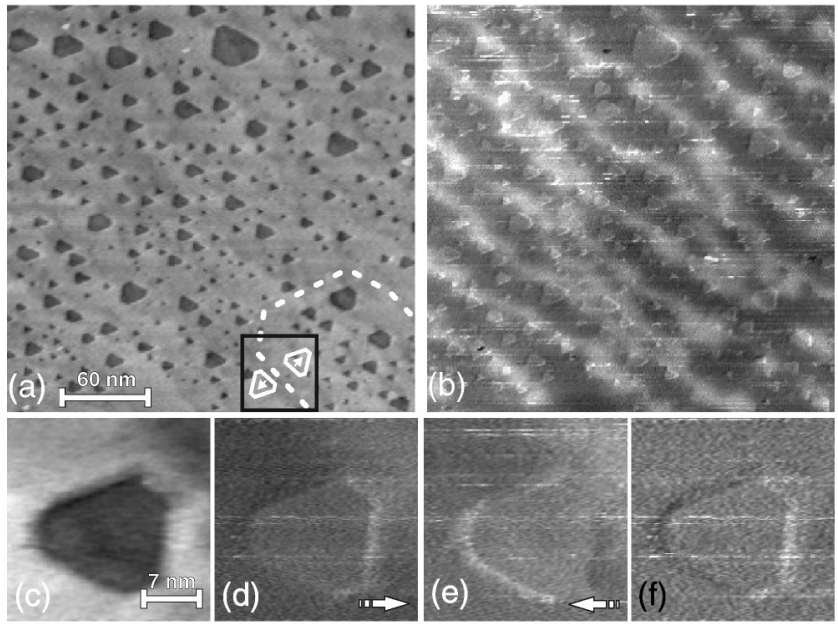

FIG. 2. $\mathrm{Bi} / \mathrm{Si}(100)-(2 \times 1)$. (a) Topography of $\mathrm{Bi} / \mathrm{Si}(100)$ $(2 \times 1)$ measured at $I_{\text {tunnel }}=50 \mathrm{pA}, U_{\text {tip }}=-1.8 \mathrm{~V}(300 \times$ $300 \mathrm{~nm}^{2}$ ). (b) Simultaneously measured BEEM image, the gray scale varies from $7 \%$ to $12 \%$ of the tunneling current. (c) Topography of a triangular shaped hole $\left(29 \times 29 \mathrm{~nm}^{2}\right)$. (d) BEEM image measured simultaneously with (c) in forward (left to right) scan direction. The gray scale varies from $8 \%$ to $15 \%$. (e) BEEM image corresponding to (c) for the reversed scan direction. (f) Tunneling current measured simultaneously with (c) and (d). The gray scale varies from 35 to $55 \mathrm{pA}$. 
contrast in 2(d) is perfectly correlated to the variation of the total tunneling current displayed in 2(f). If the tip is scanning in the upwards direction of a step there is a transient increase of the tunneling current. As the BEEM current is proportional to the tunneling current, this error signal is also found in the BEEM signal. Within the uncertainty of the experiment the ratio of BEEM current versus the total tunneling current is not enhanced or reduced. Hence, the ballistic transmission is not significantly affected by the step edges.

The results obtained with BEEM for $\mathrm{Bi} / \mathrm{Si}(111)-(7 \times 7)$ are displayed in Fig. 3. The topography in Fig. 3(a) shows a very flat $\mathrm{Bi}$ film, quite similar to $\mathrm{Si}(100)-(2 \times 1)$. Data with atomic resolution reveal an arrangement of the $\mathrm{Bi}$ atoms in a hexagonal lattice, also corroborated in LEED experiments. Although the $\mathrm{Bi}(111)$ as well as the $\mathrm{Si}(111)$ surface have a threefold symmetry, two mirror domains are observed, indicating that the Bi layer only "feels" the sixfold symmetry of the top layer of the Si surface. In the upper part of the image, the characteristic orientation of the triangles is rotated by $180^{\circ}$. Under close inspection, a domain boundary can be seen in the topography. The observations made with STM and LEED are in agreement with the work performed in other groups [26-28].

The simultaneously collected BEEM current at an injection energy of about $1.8 \mathrm{eV}$ is displayed in Fig. 3(b). The gray scale varies from $0 \%$ to $10 \%$ of the tunneling current. The almost homogeneously distributed BEEM current on most parts of the Bi plane amounts to an average value of $7 \%$. The domain boundaries appear as dark lines with a
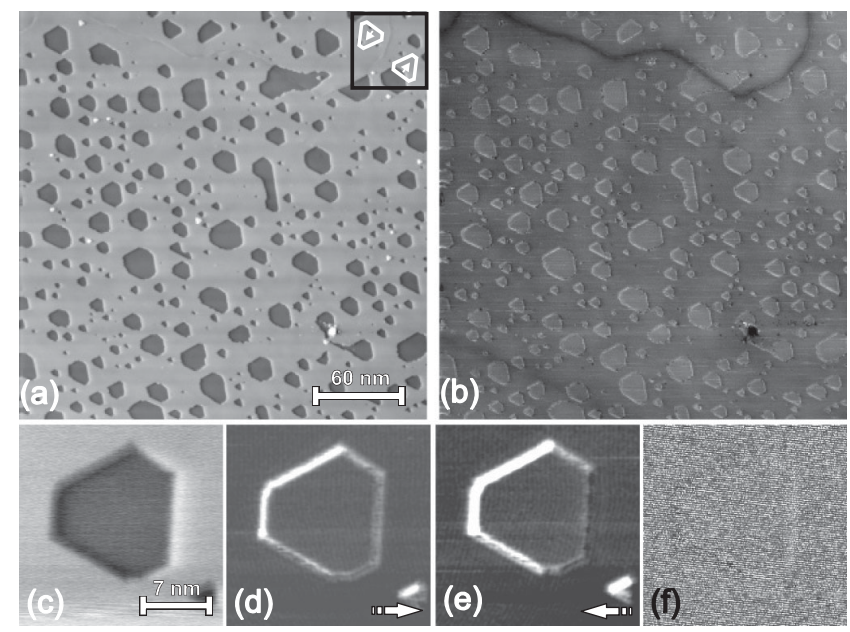

FIG. 3. $\mathrm{Bi} / \mathrm{Si}(111)-(7 \times 7)$.

(a) Topography of $\mathrm{Bi} / \mathrm{Si}(111)-(7 \times 7)$ measured at $I_{\text {tunnel }}=82 \mathrm{pA}, \quad U_{\text {tip }}=$ $-1.8 \mathrm{~V} \quad\left(300 \times 300 \mathrm{~nm}^{2}\right)$. (b) Simultaneously measured BEEM image. The gray scale varies from $0 \%$ to $10 \%$ of the tunneling current. (c) Topography of a triangular shaped hole $\left(24 \times 24 \mathrm{~nm}^{2}\right)$. (d) BEEM image measured simultaneously with (c) in forward direction. The gray scale varies from $7 \%$ to $12 \%$. (e) BEEM image corresponding to (c) for the reversed scan direction. (f) Tunneling current measured simultaneously with (c) and (d). The gray scale varies from 70 to $90 \mathrm{pA}$. reduced BEEM current. The step edges of the triangular shaped holes show a strong increase of the signal. A close view on a triangular shaped hole with its corresponding BEEM image is shown in Figs. 3(c) and 3(d). The gray scale varies from $7 \%$ to $12 \%$. The signal acquired in the opposite scan direction displayed in Fig. 3(e) is almost identical, indicating that in contrast to the faint signal on the $\mathrm{Bi} / \mathrm{Si}(100)-(2 \times 1)$ surface it is not an artifact caused by the error of the feedback loop for the tip sample distance. This is further confirmed by the image of the total tunneling current measured simultaneously with Fig. 3(d) displayed in Fig. 3(f). There is only a faint variation, which
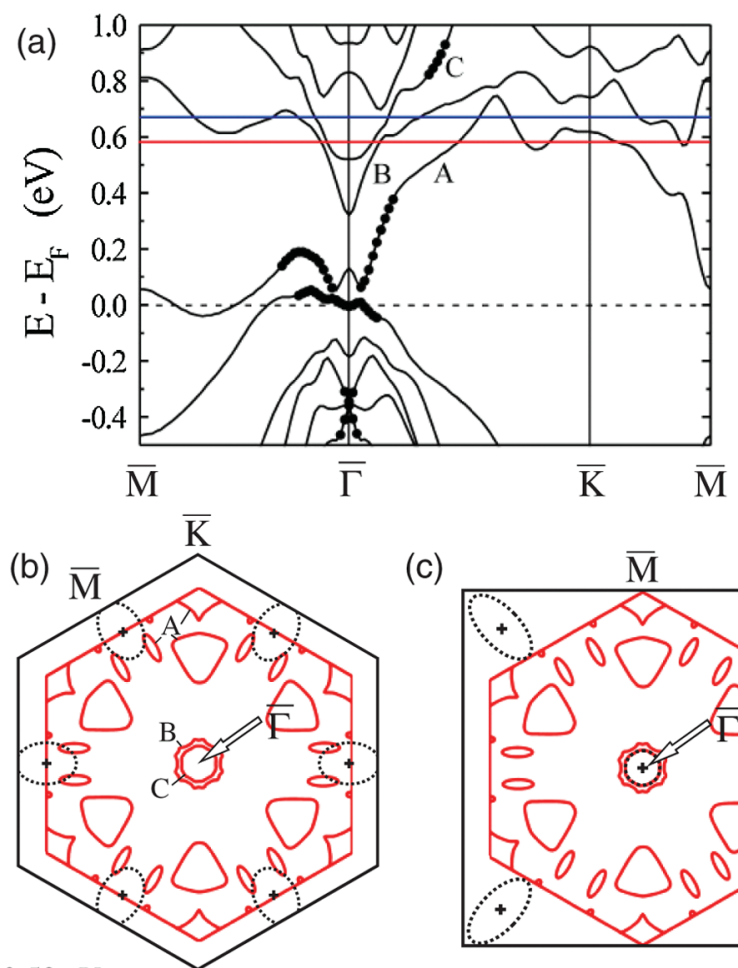

(c)
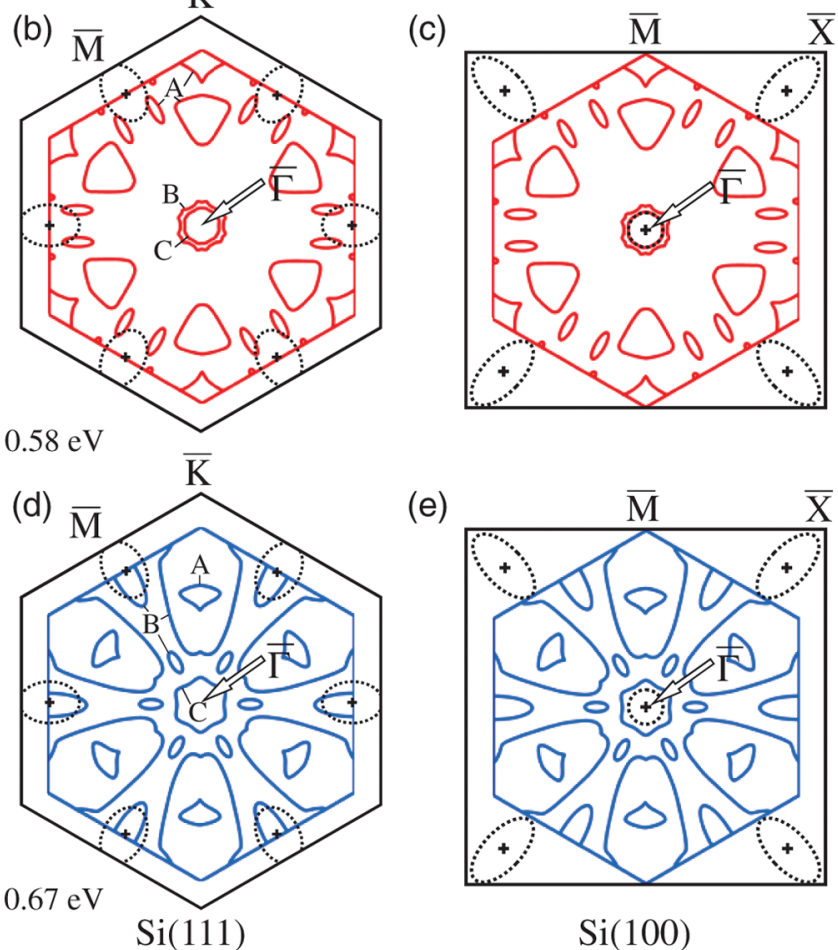

(e)

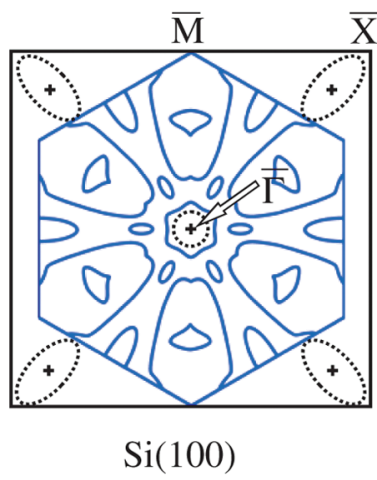

FIG. 4 (color). (a) Electronic band structure of a (freestanding) Bi film with a thickness of 5 bilayers. (b),(d) Show the projection of the electronic states of $\mathrm{Si}$ as well as $\mathrm{Bi}$ on the $\mathrm{Si}(111)$ surface. The Bi states are drawn for an energy of $0.58 \mathrm{eV}$ (red line) and $0.68 \mathrm{eV}$ (blue line), the $\mathrm{Si}$ states are pockets which are limited by the dashed line for $0.2 \mathrm{eV}$. (c),(e) Show the projection of the electronic states of $\mathrm{Si}$ as well as $\mathrm{Bi}$ on the $\mathrm{Si}(100)$ surface. 
is not correlated to the signal in the BEEM current. Very similar signals were also found at lower injection energies, e.g., at 1.3 and $1.0 \mathrm{eV}$.

To understand the different observations, the matching of the electronic band structures of the $\mathrm{Bi}$ and the $\mathrm{Si}$ at the interface has to be considered. The band structure of a thin ( 5 bilayer) Bi film has been obtained using density functional theory in the local density approximation as described in [29]. The calculations include scalar relativistic and spin-orbit coupling effects and were performed using the full-potential linearized augmented plane wave method in film geometry as implemented in the FLEUR program. The Bi film was simulated using a freestanding film, assuming overall space-inversion symmetry. Neglecting the influence of the substrate is a natural simplification in accordance with the very weak substrate overlayer interaction $[19,26]$. Figure 4(a) displays the calculated band structure for the film; the projections on the Bi(111) plane are shown in 4(b)-4(e). The red lines correspond to an electron energy of $0.58 \mathrm{eV}$, the blue lines to $0.68 \mathrm{eV}$. The data are superimposed onto the projection of the Si bulk states for the $\mathrm{Si}(111)$ and $\mathrm{Si}(100)$ planes according to [12]. For $\mathrm{Si}(100)$ [4(d) and 4(e)] the Bi state labeled by $C$ overlaps well with the Si states around $\bar{\Gamma}$. Hence, ballistic transmission from the metal to the semiconductor could be quite possible. The onset of the BEES spectrum [Fig. 1(a)] will be given by the Schottky barrier. Considering that the probability for the electrons to be injected into the metal is highest for minimal lateral momentum, the contributions for higher $k_{\|}$will be negligible. The situation is rather different for the interface to the (111) oriented Si as sketched in 4(b) and 4(c). There are no states available around $\bar{\Gamma}$ in the Si. The overlap of the electronic states occurs near the $\bar{M}$ point. However, this is only possible for an energy above about $0.64 \mathrm{eV}$. This corresponds well to the delayed onset observed in the BEES signal [Fig. 1(c)]. It also explains the increase of the BEEM signal at step edges [22,30], because the geometry of the tunneling junction favors the injection with a higher $k_{\|}$. Moreover, the less favorable overlap explains the reduction of the BEEM signal for $\mathrm{Si}(111)$ relative to $\operatorname{Si}(100)$.

In conclusion, we have shown that the differences in the BEEM current observed for $\mathrm{Bi} / \mathrm{Si}(100)-(2 \times 1)$ and $\mathrm{Bi} / \mathrm{Si}(111)-(7 \times 7)$ can be readily explained by the conservation of the lateral momentum of the electrons at the interface between the metal and the semiconductor. The delayed onset and the reduced strength of the BEEM current as a function of the electron energy for $\mathrm{Bi} / \mathrm{Si}(111)-(7 \times 7)$ compared to $\mathrm{Bi} / \mathrm{Si}(100)-(2 \times 1)$ are due to the extra lateral momentum needed for the electrons to match the band structure of the $\operatorname{Si}(111)$. Similarly, the enhancement of the BEEM current at step edges for $\mathrm{Bi}(111)$ films grown on $\mathrm{Si}(111)$ can be explained by the increased number of electrons injected from the tunneling tip into the metal film with nonzero lateral momentum.

Financial support by the Deutsche Forschungsgemeinschaft through the SFB 616 "Energy Dissipation at Surfaces" is gratefully acknowledged.

*christian.bobisch@uni-due.de

[1] W. J. Kaiser and L. D. Bell, Phys. Rev. Lett. 60, 1406 (1988).

[2] L. D. Bell and W. J. Kaiser, Phys. Rev. Lett. 61, 2368 (1988).

[3] L. J. Schowalter and E. Y. Lee, Phys. Rev. B 43, 9308 (1991).

[4] E. Y. Lee and L. J. Schowalter, J. Appl. Phys. 70, 4 (1991).

[5] M. K. Weilmeier, W.H. Rippard, and R. A. Buhrman, Phys. Rev. B 59, R2521 (1999).

[6] M.K. Weilmeier, W.H. Rippard, and R. A. Buhrman, Phys. Rev. B 61, 7161 (2000).

[7] R. Ludeke and A. Bauer Phys. Rev. Lett. 71, 1760 (1993).

[8] F. J. García-Vidal, P. L. de Andres, and F. Flores, Phys. Rev. Lett. 76, 807 (1996).

[9] L. D. Bell, Phys. Rev. Lett. 77, 3893 (1996).

[10] H. Sirringhaus, T. Meyer, E. Y. Lee, and H. von Känel, Phys. Rev. B 53, 15944 (1996).

[11] P. F. de Pablos, F. J. García-Vidal, F. Flores, and P. L. de Andres, Surf. Sci. 482-485, 430 (2001).

[12] P. L. de Andres, F. J. García-Vidal, K. Reuter, and F. Flores, Prog. Surf. Sci. 66, 3 (2001).

[13] K. Reuter et al., Phys. Rev. B 63, 205325 (2001).

[14] P. F. de Pablos, F. J. García-Vidal, F. Flores, and P. L. de Andres, Phys. Rev. B 66, 075411 (2002).

[15] W. J. Kaiser, M. H. Hecht, R. W. Fathauer, and L. D. Bell, Phys. Rev. B 44, 6546 (1991).

[16] A. Bannani, C. A. Bobisch, and R. Möller, Appl. Phys. Lett. 93, 032111 (2008).

[17] C. R. Ast and H. Höchst, Phys. Rev. Lett. 87, 177602 (2001).

[18] C. R. Ast and H. Höchst, Phys. Rev. Lett. 90, 016403 (2003).

[19] T. Hirahara et al., Phys. Rev. Lett. 97, 146803 (2006).

[20] G. Jnawali et al., Phys. Rev. B 74, 195340 (2006).

[21] A. Bannani, C. Bobisch, and R. Möller, Science 315, 1824 (2007).

[22] M. Prietsch, Phys. Rep. 253, 163 (1995).

[23] L. D. Bell and W. J. Kaiser, Annu. Rev. Mater. Sci. 26, 189 (1996).

[24] V. Narayanamurti and M. Kozhevnikov, Phys. Rep. 349, 447 (2001).

[25] G. Jnawali et al., Phys. Rev. B 76, 035337 (2007).

[26] T. Nagao et al., Phys. Rev. Lett. 93, 105501 (2004).

[27] S. Yaginuma et al., Surf. Sci. 547, L877 (2003).

[28] M. Kammler and M. Horn-von Hoegen, Surf. Sci. 576, 56 (2005).

[29] Yu. M. Koroteev, G. Bihlmayer, E. V. Chulkov, and S. Blügel, Phys. Rev. B 77, 045428 (2008).

[30] M. Dähne-Prietsch and T. Kalka, J. Electron Spectrosc. Relat. Phenom. 109, 211 (2000). 